Erschien zuerst in: Neuropsychologia 42 (2004) S. 692-697

\title{
Note \\ Cerebral lateralization in schizophrenia and dyslexia: neuromagnetic responses to auditory stimuli
}

\author{
Sabine Heim ${ }^{\mathrm{a}, \mathrm{b}, *}$, Johanna Kissler ${ }^{\mathrm{a}, 1}$, Thomas Elbert ${ }^{\mathrm{a}}$, Brigitte Rockstroh ${ }^{\mathrm{a}}$ \\ a Department of Psychology, University of Konstanz, P.O. Box D25, D-78457 Konstanz, Germany \\ ${ }^{\mathrm{b}}$ Center for Molecular and Behavioral Neuroscience, Rutgers, The State University of New Jersey, Newark, NJ 07102, USA
}

Received 16 January 2003; received in revised form 8 May 2003; accepted 16 September 2003

\begin{abstract}
Many studies have shown altered hemispheric asymmetry—particularly in perisylvian regions—in schizophrenia patients as well as in individuals with dyslexia. Here we explore the similarity of these findings comparing the localization of the magnetic auditory $\mathrm{N} 100 \mathrm{~m}$ to the German syllable [ba:] in schizophrenia patients, dyslexic adults, and healthy control subjects. Control subjects showed the typical finding of more anterior sources in the right than in the left perisylvian region. In contrast, both schizophrenia patients and dyslexic subjects displayed a symmetrical N100m source configuration. While in people with dyslexia the alteration appears to originate in the right hemisphere, left-hemispheric deviations might contribute to reduced asymmetry in schizophrenia patients. Our results indicate that an absence of lateralized auditory responses in the temporal lobes may reflect a common deviance present in dyslexia and schizophrenia. The nonspecific finding of reduced cerebral laterality may be accounted for by population-specific differences in the functional organization of perisylvian sites.
\end{abstract}

(C) 2003 Elsevier Ltd. All rights reserved.

Keywords: Auditory processing; Magnetoencephalography; N100m; Temporal cortex; Perisylvian region

\section{Introduction}

Neuroanatomical research indicates altered lateralization of the temporal plane in people with schizophrenia (Sommer, Ramsey, Kahn, Aleman, \& Bouma, 2001) and dyslexia (Shapleske, Rossell, Woodruff, \& David, 1999). In both cases pre- or perinatal neurodevelopmental abnormalities are thought to lie at the core of the problem (Akbarian et al., 1996; Galaburda, 1993).

Despite the obvious differences between the two disorders some intriguing similarities have recently been reported. For instance, an impaired perception of stop consonant-vowel syllables as usually described for children with dyslexia (Tallal, Merzenich, Miller, \& Jenkins, 1998) has also been observed in schizophrenia patients (Cienfuegos, March, Shelley, \& Javitt, 1999). In dyslexia, reduced amplitudes of the electrophysiological mismatch negativity have often been demonstrated and discussed as an index of impaired

\footnotetext{
* Corresponding author. Tel.: +49-7531-884616; fax: +49-7531-884601.

E-mail addresses: sabine.heim@uni-konstanz.de (S. Heim), johanna.kissler@uni-konstanz.de (J. Kissler).

${ }^{1}$ Co-corresponding author.
}

auditory processing (Kujala \& Näätänen, 2001). Similarly, smaller mismatch responses were recorded in schizophrenia patients in electroencephalographic (Javitt, Grochowski, Shelley, \& Ritter, 1998) as well as magnetoencephalographic (MEG) studies (Kreitschmann-Andermahr et al., 1999).

A more basic index of adequate sensory registration that is elicited by repetitive stimulation with simple tones or syllables is the auditory N100 or its magnetic counterpart, the $\mathrm{N} 100 \mathrm{~m}$. The $\mathrm{N} 100 \mathrm{~m}$ source is located more anteriorly in the right than in the left hemisphere in most normal right-handed individuals (Eulitz, Diesch, Pantev, Hampson, \& Elbert 1995; Ohtomo et al., 1998; Rockstroh et al., 2001). Several studies showed a reduction or even reversal of this asymmetry in a high proportion of schizophrenia patients (Reite, Teale, Goldstein, Whalen, \& Linnville, 1989; Rockstroh et al., 2001). In adults with dyslexia, Heim, Eulitz, and Elbert (2003a) observed lessened interhemispheric asymmetry of $\mathrm{N} 100 \mathrm{~m}$ in response to the syllable [ba:] due to an atypical source configuration in the right perisylvian region.

The analogous findings in the two different disorders give rise to questions about the underlying brain mechanisms. Recently, Crow (2000) has offered an account of schizophrenia as linked to the language development in 
humans, which may provide a unified theoretical explanation for the similarity observed. According to Crow, reduced cerebral lateralization of language fosters psychotic tendencies and thus developmental dyslexia with its altered hemispheric asymmetry might be a precursor for schizophrenia. However, to date, there is a lack of direct comparisons concerning cerebral lateralization in both disorders. If there is, in fact, a relationship between psychosis and impaired language development then similarities should be manifested in basic parameters of functional neuroanatomy known to be abnormal in these clinical populations. Here we compare the source locations of auditory N100m elicited by the syllable [ba:] in two studies: one with schizophrenia patients and the other with dyslexic adults.

\section{Methods}

In the first study (Rockstroh et al., 2001; hereafter termed "schizophrenia study"), 16 inpatients (five females; mean age $31.8 \pm 6.2$ years) with a diagnosis of either paranoid or disorganized schizophrenia and 13 healthy control subjects (four females; mean age $30.8 \pm 5.2$ years) were tested. Ten adult developmental dyslexics (four females; mean age $38.2 \pm 12.5$ years) and 10 normal controls (five females; mean age $32.8 \pm 13.2$ years) participated in the second study (Heim et al., 2003a; hereafter termed "dyslexia study"). All subjects were native speakers of German and had finished the minimum of formal education (9 years in Germany). The groups did not differ significantly in terms of age and handedness. All schizophrenia patients were under neuroleptic medication. Dyslexic and control subjects were free of psychiatric disorders requiring consultation (present or past) and any psychotropic medication. Participants had no history of neurological disease and evidenced normal hearing thresholds. Informed consent was obtained from each subject (cf. Declaration of Helsinki); all were paid for their participation.

In both experiments, magnetic brain activity in response to the syllable [ba:] was recorded by means of a 148-channel whole-head neuromagnetometer (BTi, MAGNES $2500^{\mathrm{TM}}$, 4D Neuroimaging, San Diego, CA, USA). The total stimulus duration was $250 \mathrm{~ms}$, stimulus intensity was at $60 \mathrm{~dB}$ sensation level. The schizophrenia study consisted of a passive listening task. Series of syllables were presented through ear tubes either to the subject's left or right ear while resting in a supine position. The intertrial interval (stimulus onset to onset) varied pseudorandomly from 2.7 to $3.3 \mathrm{~s}$. In the dyslexia study, passive listening to the standard syllable [ba:] $(P=$ 0.8 ) was also required during an oddball paradigm. Syllables were delivered binaurally to seated participants with a constant intertrial interval of $1 \mathrm{~s}$.

While the schizophrenia study was designed to examine solely the N100m component, the dyslexia study is part of our ongoing work in which we are also interested in evaluating the mismatch field. To account for possible differences in task design, we compared N100m sources between the controls of both studies (see also Sections 3 and 4).

For subjects of both studies, sources of the auditory neuronal activity were estimated by determining the equivalent current dipole around the N100m root mean square maximum using 34-37 channels separately over the left and right supratemporal cortices. The following constraints were placed on the dipole fits a priori: (a) response peak within the first $150 \mathrm{~ms}$ post-stimulus onset; (b) averages based at least on 80 artifact-free trials (artifact rejection threshold: signal variations $>5 \mathrm{pT}$ in the MEG or $>120 \mu \mathrm{V}$ in the electro-oculogram); (c) goodness of fit $>90 \%$; (d) distance of equivalent current dipole to midsagittal plane $>2.5 \mathrm{~cm}$; (e) inferior-superior value $>3$ and $<8 \mathrm{~cm}$; (f) equivalent current dipole oriented downwards indexing the negativity of the component. The confidence volume did not differ between subject groups; the average value was $213.5 \mathrm{~mm}^{3}$.

\section{Results}

In all participants the $\mathrm{N} 100 \mathrm{~m}$ component was evident in the latency range between 80 and $145 \mathrm{~ms}$ after syllable onset. $^{2}$ Fig. 1 exemplifies magnetic waveforms and field distributions of $\mathrm{N} 100 \mathrm{~m}$ in individual subjects. At first glance the field contours of the control and schizophrenia subject look more alike than the contours of the particular control and dyslexia subject. This may reflect differences in head size or position, which can affect the amplitudes as depicted in Fig. 1, but have no influence on source locations.

Source locations of $\mathrm{N} 100 \mathrm{~m}$ were compared in mixedmodel analyses of variance with group (schizophrenia, dyslexic, control/schizophrenia study, control/dyslexia study) as between-subjects factor and hemisphere (left, right) as within-subjects factor.

Results revealed a significant group $\times$ hemisphere interaction for source localizations along the anterior-posterior axis $(F(3,45)=5.7, P<0.01$; see Fig. 2 A and B). While healthy subjects of both studies displayed the typical asymmetrical localization with more anterior $\mathrm{N} 100 \mathrm{~m}$ sources in the right than in the left hemisphere, the clinical groups had altered, more symmetrical source positions. In dyslexic subjects, deviances in laterality were associated with an atypical source configuration in the right hemisphere $(t 18=-2.2$, $P<0.05$ ), whereas schizophrenia patients tended to show left-hemisphere differences $(t 27=-1.7, P<0.1)$.

No differences in source positions along the medial-lateral axis were observed (Fig. 2C). Analysis of the inferiorsuperior coordinates yielded a significant group $\times$ hemisphere interaction $(F(3,45)=3.1, P<0.05$; see Fig. 2D). However, clinical versus control group contrasts of

\footnotetext{
${ }^{2}$ Since contralateral responses in participants of the schizophrenia study were significantly faster than binaural responses in subjects of the dyslexia study $(F(3,45)=11.8, P<0.001)$, ipsilateral data from the schizophrenia study were chosen for analysis $(F(3,45)=0.4, P<0.72)$.
} 


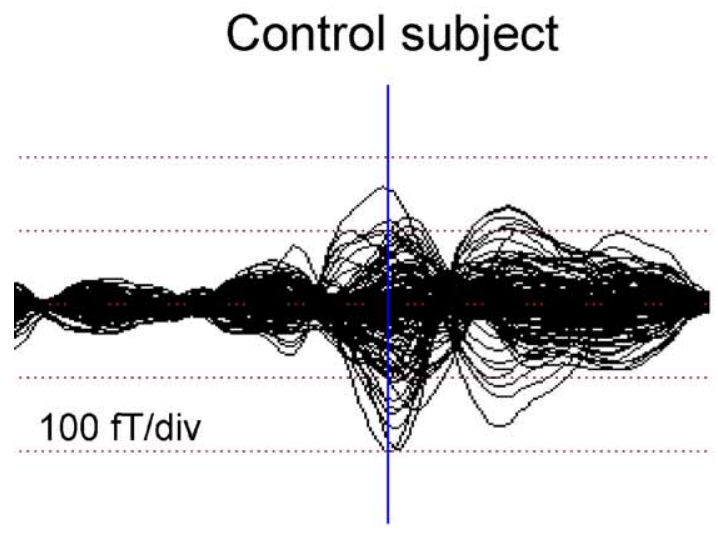

$119 \mathrm{~ms}$

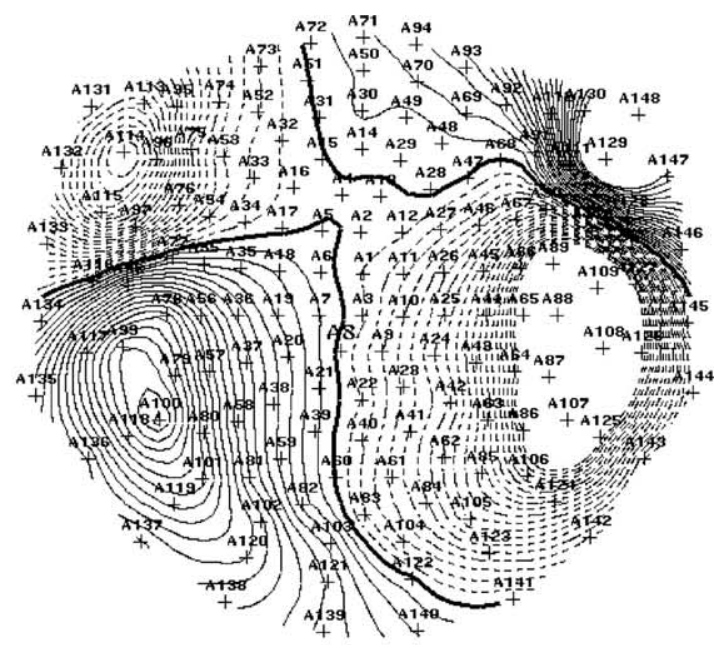

\section{Schizophrenia patient}

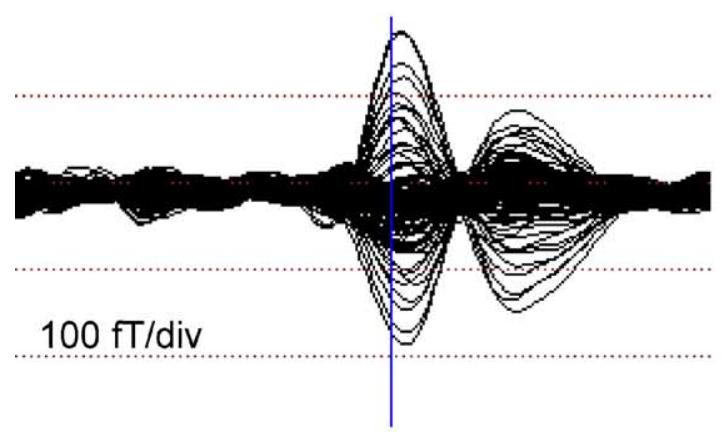

$119 \mathrm{~ms}$

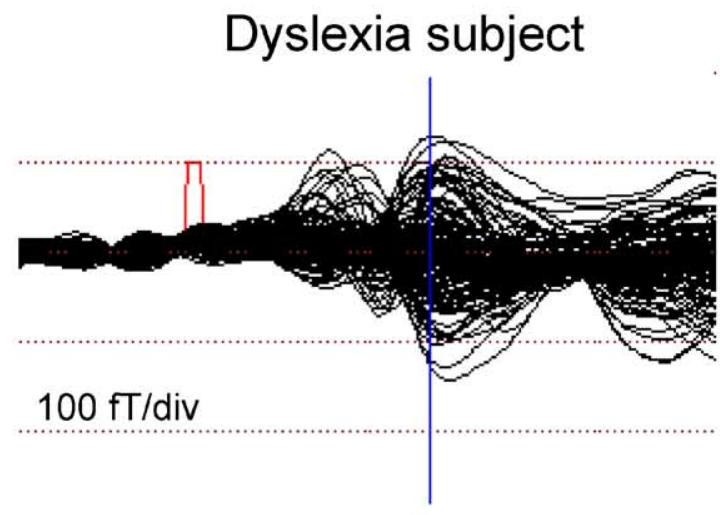

$122 \mathrm{~ms}$

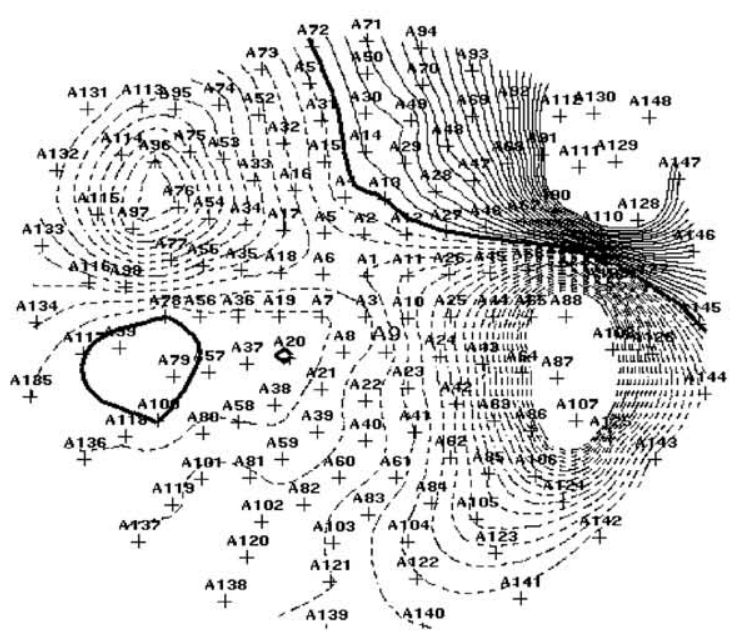

Nasion

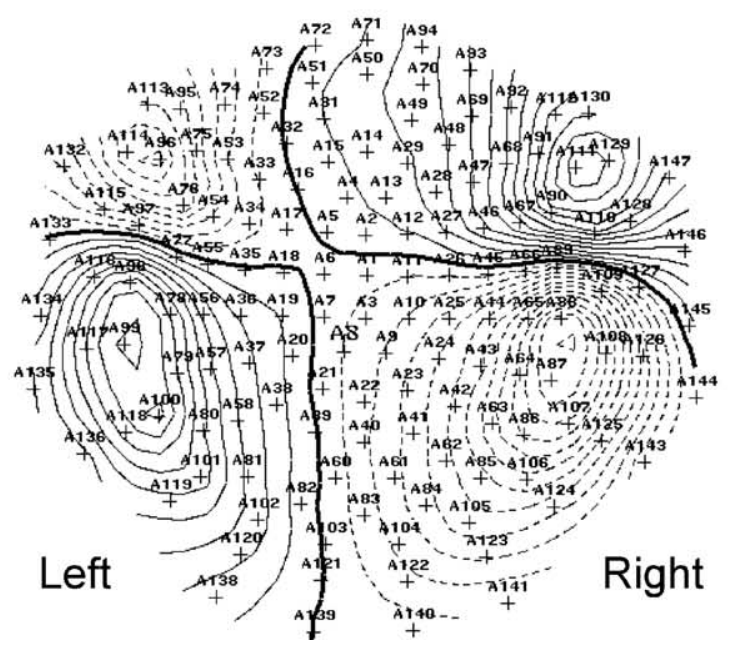

Fig. 1. Averaged neuromagnetic responses (overlay of all 148 channels) to the syllable [ba:] in an individual control subject, an individual schizophrenia patient, and an individual dyslexic subject. Vertical lines indicate latencies following stimulus onset (bar) for the best dipole fit of N100m in each subject (left). Corresponding isofield contour maps at the respective latencies of the component N100m. Solid contour lines depict the outgoing, the dashed lines the ingoing magnetic field, and the thick solid line zero flux. The spacing of the contours is $10 \mathrm{ft}$. A1-A148 represent the channel numbers (right). 


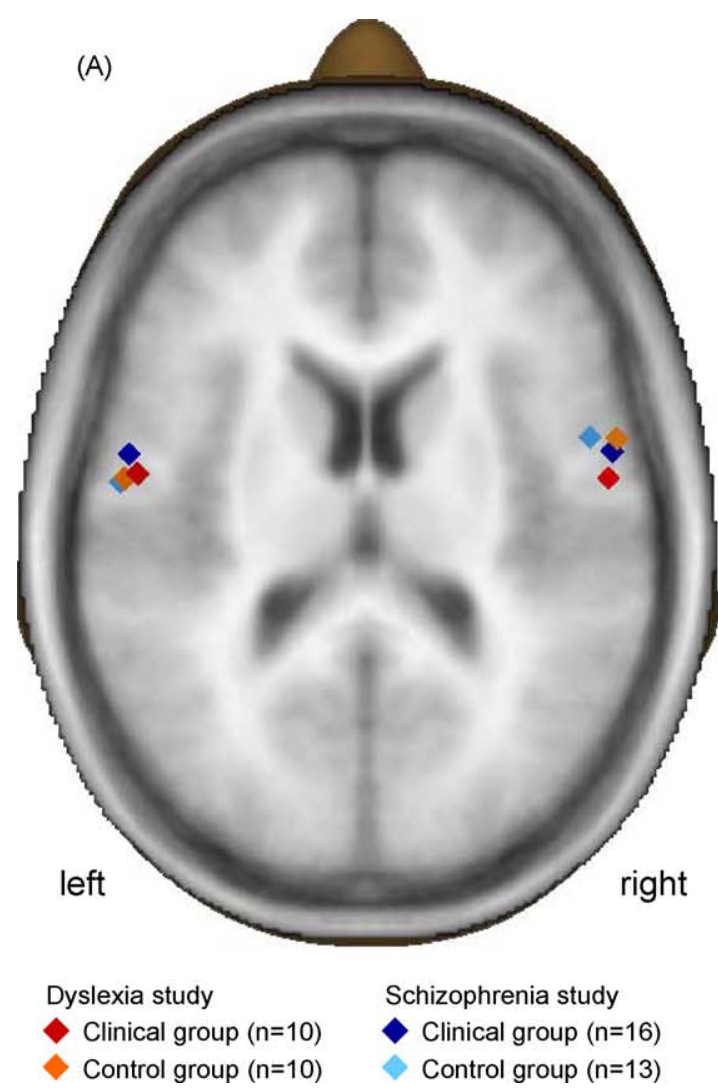

(B)

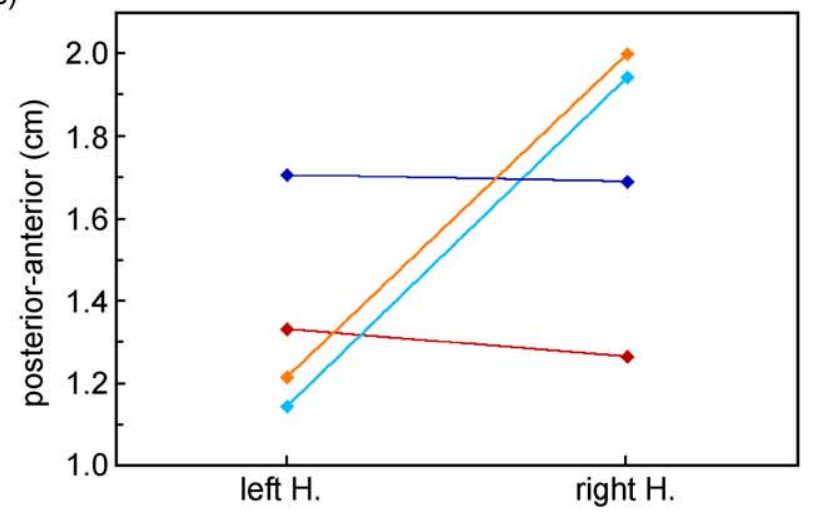

(C)

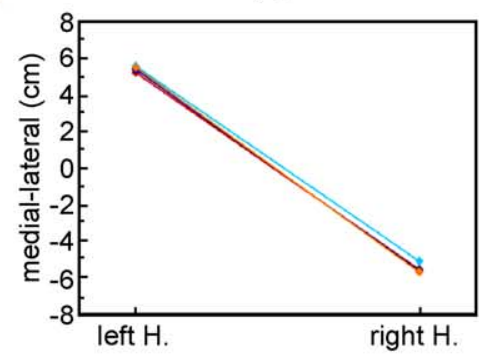

(D)

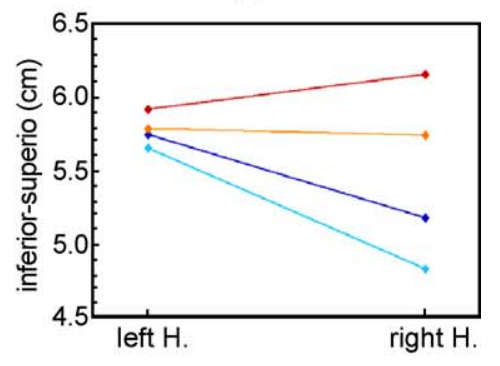

Fig. 2. (A) Mean source locations of the N100m for participants of the schizophrenia study (red and orange) and dyslexia study (dark and light blue) superimposed on an axial slice of the Montreal Brain. Source localizations along the posterior-anterior (B, see also A); medial-lateral (C); and inferior-superior (D) axes. Note the atypical hemispheric asymmetry in both schizophrenia patients and dyslexic individuals on the posterior-anterior plane (A and B). H. denotes hemisphere.

inferior-superior values within the studies did not reach statistical significance.

\section{Discussion}

The current report targeted similarities and differences of cerebral lateralization in schizophrenia and dyslexia. Using magnetic source imaging, we evaluated the localizations of the auditory N100m in response to the speech stimulus [ba:]. The two clinical groups failed to demonstrate the hemispheric asymmetry observed in healthy controls. While in unimpaired individuals the N100m source was located more anterior in the right perisylvian region than in the left, both schizophrenia patients and dyslexic subjects showed symmetrical source positions between the hemispheres. However, the N100m tended to be localized more anteriorly in schizophrenia patients than in dyslexic participants. Closer inspection of the data revealed that in dyslexia, the deviation in cerebral lateralization was associated with an atypical source location in the right hemisphere, whereas in schizophrenia patients a shift in left-hemisphere source configurations appears to contribute to the altered hemispheric asymmetry.
The sources of the N100m, which is thought to index basic sensory registration, have been localized to Heschl's gyrus and the planum temporale (Godey, Schwartz, de Graaf, Chauvel, \& Liegeois-Chauvel, 2001). The planum temporale is a triangular landmark situated on the supratemporal surface just posterior to the first Heschl's gyrus, inside the Sylvian fissure. The left planum coincides with part of Wernicke's speech comprehension area (Shapleske et al., 1999). Large post-mortem studies (Geschwind \& Levitsky, 1968; Wada, Clarke, \& Hamm, 1975) each including 100 normal adult brains found that it was symmetrically sized between the hemispheres in $16 \%$, whereas $10.5 \%$ showed a rightward asymmetry and $73.5 \%$ a leftward. The planum temporale is thought to be an important substrate of left-hemispheric language lateralization (Geschwind \& Levitsky, 1968; Wada et al., 1975).

Our unimpaired subjects displayed the typical, across experiments virtually identical right-left asymmetry of N100m. This functional asymmetry has been discussed against the background of two neuroanatomical hypotheses. (i) Crow (1997) assumes that 'cerebral torque' results from an anatomical asymmetry in the size of occipital structures, causing a forward shift of the right-hemisphere temporal lobe. (ii) According to Binder, Frost, Hammeke, Rao, and 
Cox (1996), larger frontal and peri-rolandic mass on the left side pushes the point of upward deflection of the Sylvian fissure posteriorly and tilts the planum temporale back. Conversely, larger parietal cortex on the right side might push the point of sylvian deflection anteriorly and tilt the planum forward.

To what extent the more symmetrical organization in the clinical populations is caused by such anatomical alterations or rather functional deviances remains unclear at present. Less $\mathrm{N} 100 \mathrm{~m}$ lateralization might reflect altered neuronal morphology in temporal-plane sites of dyslexic individuals (Galaburda, 1993). It is conceivable that based on these alterations other (right posterior) perisylvian regions become involved in auditory processing. These substituted regions may not perform the task as efficiently as a normally developed planum temporale would. A similar mechanism albeit in the left hemisphere might be at work in schizophrenia.

Deviations in hemispheric asymmetry have often been viewed as a consequence of genetic mechanisms in both schizophrenia and dyslexia (Annett, 1998). Traditionally, this account implies life-long stability of the trait in question. Several studies in animals and humans, however, have demonstrated that central nervous system mechanisms can be modified by experience (Elbert, Heim, \& Rockstroh, 2001). Experience-related changes in auditory cortex appear in response to a heavy training schedule realized in a behaviorally relevant context (Elbert \& Heim, 2001). Recently, Heim, Eulitz, and Elbert (2001) showed that improvements in language-related tests following training are mirrored in altered magnetic brain activity in children with literacy problems. Although before intervention children revealed a predominantly right-hemisphere activation pattern, post-training evaluations indicated left-lateralized responses similar to those found in normally developing age-mates. Similar results have been reported by Simos et al. (2002). Such findings are promising as they indicate that hemispheric balance in general can be affected by experiential factors. The importance of state variables paralleling cerebral lateralization is also supported in schizophrenia patients (Rockstroh et al., 2001). Rockstroh and colleagues have previously shown that patients who had higher negative symptoms scores displayed decreased laterality indices with respect to the magnitude of dipole activation around $100 \mathrm{~ms}$ after stimulus onset.

One limitation of the present report is that data from two different studies were used. Therefore we compared source localizations of $\mathrm{N} 100 \mathrm{~m}$ between the control groups of the two studies. Results revealed that the striking similarities in N100m sources across healthy subjects from both studies justify the comparison: Localizations on the medial-lateral plane did not differ at all either between unimpaired individuals or clinical groups. Importantly, no differences between healthy subjects from either study were observed on the anterior-posterior plane, where differences between clinical and normal samples have previously been reported. Although source localizations in the inferior-superior direc- tion varied across studies implying a certain influence of task, there were no clinical-normative differences within the studies regarding the parameter under consideration.

In future, studies involving larger sample sizes and allowing for the differential assessment of different subtypes of both dyslexia and schizophrenia would be desirable. The influence of handedness which was controlled for in the current studies but which is generally an important factor affecting brain lateralization (Annett, 1998) should also be systematically assessed in both of the present disorders.

Finally, the possibility that several brain processes contribute to the N100m peak (Reite, Teale, Rojas, \& McCormick, 2002) should be considered in future experiments. Conceivably, different subprocesses are disturbed in various disorders.

\section{Clinical implications}

With its high temporal and spatial resolution, whole-head MEG technique provides a sensitive tool for examining cerebral laterality in clinical as well as normal populations. Moreover, MEG is non-invasive and unobtrusive, thus it is well suited for studying people, who often have a prolonged history of medical and psychological testing.

Environmental factors can influence functional brain architecture: training programs implemented in children with literacy problems may be capable of altering cortical organization (Heim et al., 2001; Simos et al., 2002). The capacities and limits of intervention methods should be evaluated in disorders encompassing the language domain regarding both their behavioral and neural effects. In schizophrenia, the possibility that symptomatic change is correlated with subtle alterations in cerebral laterality is worth exploring. Albeit patterns of hemispheric asymmetry are assumed to be under genetic control, there is growing evidence of state-dependent variations in neuronal functioning (Elbert \& Heim, 2001; Elbert et al., 2001).

Although there are similar abnormalities in cerebral lateralization, this does not necessarily imply a common underlying mechanism of the two symptomatically so different disorders. While schizophrenia may well be related to the human capacity for language as suggested by Crow (2000) and evidenced by delayed language development in a fair proportion of patients (DeLisi, 2001), identification of the affected language domain is indispensable. Both language problems and alterations in cerebral asymmetry could result from various, possibly unspecific reasons. One promising approach to reveal differences in interhemispheric asymmetry is to directly compare brain responses between schizophrenia patients and dyslexic individuals. Indeed, closer scrutiny of the present data suggests population-specific patterns of alteration-predominantly right-hemispheric in dyslexia, possibly left-hemispheric in schizophrenia consistent with other reports (e.g., Heim, Eulitz, \& Elbert, 2003b; Reite et al., 1989). In order to 
enhance the clinical significance of findings as the present one, it is crucial to uncover population-specific patterns of cortical organization.

\section{Acknowledgements}

Research was supported by the Deutsche Forschungsgemeinschaft and the Volkswagen-Stiftung.

\section{References}

Akbarian, S., Kim, J. J., Potkin, S. G., Hetrick, W. P., Bunney Jr., W. E., \& Jones, E. G. (1996). Maldistribution of interstitial neurons in prefrontal white matter of the brains of schizophrenic patients. Archives of General Psychiatry, 53, 425-436.

Annett, M. (1998). Handedness and cerebral dominance: The right shift theory. Journal of Neuropsychiatry and Clinical Neuroscience, 10, 459-469.

Binder, J. R., Frost, J. A., Hammeke, T. A., Rao, S. M., \& Cox, R. W. (1996). Function of the left planum temporale in auditory and linguistic processing. Brain, 119, 1239-1247.

Cienfuegos, A., March, L., Shelley, A. M., \& Javitt, D. C. (1999). Impaired categorical perception of synthetic speech sounds in schizophrenia. Biological Psychiatry, 45, 82-88.

Crow, T. J. (1997). Schizophrenia as failure of hemispheric dominance for language. Trends in Neuroscience, 20, 339-343.

Crow, T. J. (2000). Schizophrenia as the price that Homo sapiens pays for language: A resolution of the central paradox in the origin of the species. Brain Research Reviews, 31, 118-129.

DeLisi, L. E. (2001). Speech disorder in schizophrenia: Review of the literature and exploration of its relation to the uniquely human capacity for language. Schizophrenia Bulletin, 27, 481-496.

Elbert, T., \& Heim, S. (2001). Cortical reorganization: A light and a dark side. Nature, 411, 139.

Elbert, T., Heim, S., \& Rockstroh, B. (2001). Neural plasticity and development. In C. Nelson, \& M. Luciana (Eds.), Handbook of developmental cognitive neuroscience (pp. 191-202). Cambridge, MA: MIT Press.

Eulitz, C., Diesch, E., Pantev, C., Hampson, S., \& Elbert, T. (1995). Magnetic and electric brain activity evoked by the processing of tone and vowel stimuli. Journal of Neuroscience, 15, 2748-2755.

Galaburda, A. M. (1993). Neuroanatomical basis of developmental dyslexia. Neurologic Clinics, 11, 161-173.

Geschwind, N., \& Levitsky, W. (1968). Human brain: Left-right asymmetries in temporal speech region. Science, 161, 186-187.

Godey, B., Schwartz, D., de Graaf, J. B., Chauvel, P., \& Liegeois-Chauvel, C. (2001). Neuromagnetic source localization of auditory evoked fields and intracerebral evoked potentials: A comparison of data in the same patients. Clinical Neurophysiology, 112, 1850-1859.

Heim, S., Eulitz, C., \& Elbert, T. (2001). Effects of syllabic training on phonological processing and cortical organization in children with specific language impairment. Society for Neuroscience Abstracts, 27, Program No. 447.17.

Heim, S., Eulitz, C., \& Elbert, T. (2003a). Altered hemispheric asymmetry of auditory $\mathrm{N} 100 \mathrm{~m}$ in adults with developmental dyslexia. NeuroReport, 14, 501-504.

Heim, S., Eulitz, C., \& Elbert, T. (2003b). Altered hemispheric asymmetry of auditory P100m in dyslexia. European Journal of Neuroscience, 17, 1715-1722.

Javitt, D. C., Grochowski, S., Shelley, A. M., \& Ritter, W. (1998). Impaired mismatch negativity (MMN) generation in schizophrenia as a function of stimulus deviance, probability, and interstimulus/interdeviant interval. Electroencephalography and Clinical Neurophysiology, 108, 143-153.

Kreitschmann-Andermahr, I., Rosburg, T., Meier, T., Volz, H. P., Nowak, H., \& Sauer, H. (1999). Impaired sensory processing in male patients with schizophrenia: A magnetoencephalographic study of auditory mismatch detection. Schizophrenia Research, 35, 121-129.

Kujala, T., \& Näätänen, R. (2001). The mismatch negativity in evaluating central auditory dysfunction in dyslexia. Neuroscience and Biobehavioral Reviews, 25, 535-543.

Ohtomo, S., Nakasato, N., Kanno, A., Hatanaka, K., Shirane, R., Mizoi, K., \& Yoshimoto, T. (1998). Hemispheric asymmetry of the auditory evoked $\mathrm{N} 100 \mathrm{~m}$ response in relation to the crossing point between the central sulcus and Sylvian fissure. Electroencephalography and Clinical Neurophysiology, 108, 219-225.

Reite, M., Teale, P., Goldstein, L., Whalen, J., \& Linnville, S. (1989). Late auditory magnetic sources may differ in the left hemisphere of schizophrenic patients. A preliminary report. Archives of General Psychiatry, 46, 565-572.

Reite, M., Teale, P., Rojas, D. C., \& McCormick, K. H. (2002). MEG findings of altered brain laterality in schizophrenia: A critical review. In H. Nowak, J. Haueisen, F. Gießler, \& R. Huonker (Eds.), Proceedings of the 13th International Conference on Biomagnetism, Jena, Germany (p. 207). Berlin: VDE Verlag.

Rockstroh, B., Kissler, J., Mohr, B., Eulitz, C., Lommen, U., Wienbruch, C., Cohen, R., \& Elbert, T. (2001). Altered hemispheric asymmetry of auditory magnetic fields to tones and syllables in schizophrenia. Biological Psychiatry, 49, 694-703.

Shapleske, J., Rossell, S. L., Woodruff, P. W., \& David, A. S. (1999). The planum temporale: A systematic, quantitative review of its structural, functional and clinical significance. Brain Research Reviews, 29, 26 49.

Simos, P. G., Fletcher, J. M., Bergman, E., Breier, J. I., Foorman, B. R., Castillo, E. M., Davis, R. N., Fitzgerald, M., \& Papanicolaou, A. C. (2002). Dyslexia-specific brain activation profile becomes normal following successful remedial training. Neurology, 58, 1203-1213.

Sommer, I., Ramsey, N., Kahn, R., Aleman, A., \& Bouma, A. (2001). Handedness, language lateralisation and anatomical asymmetry in schizophrenia: Meta-analysis. British Journal of Psychiatry, 178, 344-351.

Tallal, P., Merzenich, M. M., Miller, S., \& Jenkins, W. (1998). Language learning impairments: Integrating basic science, technology, and remediation. Experimental Brain Research, 123, 210-219.

Wada, J. A., Clarke, R., \& Hamm, A. (1975). Cerebral hemispheric asymmetry in humans. Cortical speech zones in 100 adults and 100 infant brains. Archives of Neurology, 32, 239-246. 